\title{
Notch signaling is essential for vascular morphogenesis in mice
}

\author{
Luke T. Krebs, ${ }^{1}$ Yingzi Xue, ${ }^{1,5}$ Christine R. Norton, ${ }^{1}$ John R. Shutter, ${ }^{2}$ Maureen Maguire,,${ }^{1,6}$ \\ John P. Sundberg, ${ }^{1}$ Daniel Gallahan, ${ }^{3}$ Violaine Closson, ${ }^{4}$ Jan Kitajewski, ${ }^{4}$ Robert Callahan, ${ }^{3}$ \\ Gilbert H. Smith, ${ }^{3}$ Kevin L. Stark, ${ }^{2}$ and Thomas Gridley ${ }^{1,7}$ \\ ${ }^{1}$ The Jackson Laboratory, Bar Harbor, Maine 04609 USA; ${ }^{2}$ Department of Molecular Genetics, Amgen, Inc., Thousand Oaks,
California 91320 USA; ${ }^{3}$ Laboratory of Tumor Immunology and Biology, National Cancer Institute, National Institutes
of Health (NIH), Bethesda, Maryland 20892 USA; ${ }^{4}$ Department of Pathology and Obstetrics and Gynecology, Columbia University,
College of Physicians and Surgeons, New York, New York 10032 USA
}

The Notch gene family encodes large transmembrane receptors that are components of an evolutionarily conserved intercellular signaling mechanism. To assess the role of the Notch4 gene, we generated Notch4-deficient mice by gene targeting. Embryos homozygous for this mutation developed normally, and homozygous mutant adults were viable and fertile. However, the Notch4 mutation displayed genetic interactions with a targeted mutation of the related Notch1 gene. Embryos homozygous for mutations of both the Notch4 and Notch1 genes often displayed a more severe phenotype than Notch1 homozygous mutant embryos. Both Notch1 mutant and Notch1/Notch4 double mutant embryos displayed severe defects in angiogenic vascular remodeling. Analysis of the expression patterns of genes encoding ligands for Notch family receptors indicated that only the $\mathbf{D 1 1 4}$ gene is expressed in a pattern consistent with that expected for a gene encoding a ligand for the Notch1 and Notch4 receptors in the early embryonic vasculature. These results reveal an essential role for the Notch signaling pathway in regulating embryonic vascular morphogenesis and remodeling, and indicate that whereas the Notch4 gene is not essential during embryonic development, the Notch4 and Notch1 genes have partially overlapping roles during embryogenesis in mice.

[Key Words: Notch signaling pathway; angiogenesis; vasculogenesis; ephrins]

Received December 16, 1999; revised version accepted March 23, 2000.

The Notch signaling pathway is an evolutionarily conserved intercellular signaling mechanism, and mutations in its components disrupt cell fate specification and embryonic development in organisms as diverse as insects, nematodes, and mammals. This signaling pathway was first studied in Drosophila. The Notch gene of Drosophila encodes a large transmembrane receptor that, at the extracellular surface of a cell, interacts with membrane-bound ligands encoded by the Delta and Serrate genes. The signal induced by ligand binding is then transmitted at the intracellular surface in a process involving proteolytic processing of the receptor and nuclear translocation of the intracellular domain of the Notch protein (for recent reviews of the Notch signaling pathway, see Gridley 1997; Weinmaster 1997; Chan and Jan 1998; Greenwald 1998; Artavanis-Tsakonas et al. 1999).

Four Notch genes have been described in mice. The Notch 4 gene was originally identified as a common viral

Present addresses: ${ }^{5}$ Skirball Institute of Bimolecular Medicine, New York University Medical Center, New York, New York 10016 USA; ${ }^{6}$ Schering-Plogh Research Institute, Kenilworth, New Jersey 07033 USA. ${ }^{7}$ Corresponding author.

E-MAIL gridley@jax.org; FAX 207-288-6077. integration site (originally termed the int3 locus) in mouse mammary tumor virus (MMTV)-induced mammary tumors (Gallahan and Callahan 1987; Robbins et al. 1992). In these tumors, proviral integration leads to the production of a truncated Notch 4 transcript. This truncated transcript initiates within the 3' MMTV long terminal repeat and includes the region encoding the intracellular domain of the Notch4 protein (Robbins et al. 1992; Gallahan and Callahan 1997), which is a constitutively active form of the Notch4 protein. Further work in transgenic mouse models and in tissue culture cells has demonstrated that unregulated expression of the cytoplasmic domain of the Notch4 protein inhibits mammary development (Smith et al. 1995; Uyttendaele et al. 1998). Cloning of the full-length Notch4 cDNA revealed that it encodes a protein of $200 \mathrm{kD}$ with fewer epidermal growth factor (EGF)-like repeats and a shorter intracellular domain than the other mammalian Notch homologs (Uyttendaele et al. 1996; Gallahan and Callahan 1997; Shirayoshi et al. 1997). Somewhat surprisingly, expression analyses indicated that embryonic expression of the Notch4 gene was largely restricted to vascular endothelial cells (Uyttendaele et al. 1996; Shirayoshi et al. 1997). 
Formation of the vascular system is one of the earliest and most important events during embryogenesis in mammals. During the early stages of vascular development in both the mammalian embryo and its extraembryonic membranes such as the yolk sac, endothelial cell precursors differentiate and coalesce into a network of homogeneously sized primitive blood vessels (the primary vascular plexus) in a process termed vasculogenesis (Risau and Flamme 1995). This primary vascular plexus is then remodeled by the process of angiogenesis, which involves the sprouting, branching, splitting, and differential growth of vessels in the primary plexus to form both the large and small vessels of the mature vascular system (Risau 1997; Gale and Yancopoulos 1999). A number of different intercellular signaling pathways have been implicated in the control of vasculogenesis and angiogenesis in mammals. These pathways include the vascular endothelial growth factor pathway, the transforming growth factor- $\beta$ (TGF- $\beta$ ) pathway, the angiopoietin/Tie receptor pathway, and the ephrin/Eph receptor pathway (for reviews, see Folkman and D'Amore 1996; Hanahan 1997; Carmeliet and Collen 1999; Gale and Yancopoulos 1999; Neufeld et al. 1999).

A number of observations indicate that the Notch signaling pathway also plays a critical role in vascular development and homeostasis. In addition to the Notch4 gene, the Notch1 gene is expressed in endothelial cells in the embryonic vasculature (Franco del Amo et al. 1992; Reaume et al. 1992). Analysis of targeted mutations of genes encoding Notch ligands has shown that both Jag1 (Xue et al. 1999) and Dll1 (Hrabé de Angelis et al. 1997) homozygous mutant embryos die from vascular defects and hemorrhaging at approximately gestational day E10.5. Expression of the human JAG1 gene is induced in an in vitro angiogenesis model, and administration of Jag1 antisense oligonucleotides modulates in vitro angiogenesis (Zimrin et al. 1996). In adults, a role for the Notch pathway in vascular homeostasis has been suggested by the finding that the degenerative vascular disease CADASIL is caused by missense mutations in the Notch3 gene (Joutel et al. 1996).

In this study we examined whether the Notch4 gene plays an essential role during embryonic development in mice. Notch4-deficient mice were viable and fertile and exhibited no obvious mutant phenotype. However, the Notch4 null mutation genetically interacted with a mutation we had constructed previously in the Notch1 gene (Swiatek et al. 1994), indicating partial functional redundancy between the Notch1 and Notch4 genes during embryogenesis. Further characterization of the phenotypes

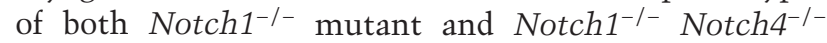
double mutant embryos revealed extensive defects in angiogenic vascular remodeling that affected the embryo, the yolk sac, and the placenta. Characterization of the expression patterns of the genes encoding Notch ligands revealed that only the newly isolated D114 gene (Shutter et al. 2000) was expressed in a pattern consistent with that expected for a gene encoding a ligand for the Notch1 and Notch4 receptors in the early embryonic vasculature.

\section{Results}

Mice homozygous for a targeted mutation of the Notch4 gene are viable and fertile

To analyze the role of the Notch4 gene, we created a deletion allele by gene targeting. A Notch4 targeting vector was constructed that deleted a $1.0-\mathrm{kb}$ genomic fragment containing exons 21 and 22 encoding part of the extracellular domain of the protein (Fig. 1A). We refer to this allele as Notch4 ${ }^{d 1}$. Germ-line transmission of the Notch $4^{d 1}$ mutant allele was obtained for two targeted clones (Fig. 1B). Mice heterozygous for the Notch $4^{d 1}$ mutation appeared normal and were fertile.

To examine whether mice homozygous for the Notch $4^{d 1}$ mutation were viable, heterozygous $\mathrm{F}_{1}$ animals were intercrossed and the genotypes of $F_{2}$ progeny were determined 2-3 weeks after birth. Mice homozygous for the Notch $4^{d 1}$ mutation (designated Notch4 ${ }^{-/-}$) were recovered at the expected frequency and by morphological and histological analyses did not differ from heterozygous and wild-type littermates. Genomic PCR analysis confirmed that the expected genomic fragment was deleted in the Notch4 $4^{-/-}$mutant homozygotes (Fig. 1C). Mammary glands from the Notch4 ${ }^{-/-}$mutant homozygotes were examined in particular detail, because the Notch4 gene was initially identified as the int3 gene, a common integration site for MMTV-induced mammary tumors (Robbins et al. 1992), and unregulated expression of the cytoplasmic domain of the Notch4 protein inhibits mammary development (Smith et al. 1995; Uyttendaele et al. 1998). However, morphological and histological analyses of mammary glands isolated from virgin, pregnant, and lactating females did not reveal any defects in the Notch4 ${ }^{-/-}$homozygotes (data not shown). We analyzed RNA isolated from Notch4 ${ }^{-/-}$homozygous mutant adult animals for the presence of Notch4 transcripts by RT-PCR with several different primer sets (Fig. 1D), and analyzed Notch4 ${ }^{-/-}$homozygous mutant embryos by whole-mount in situ hybridization with a Notch 4 antisense riboprobe encoding the entire intracellular domain of the Notch4 protein (Fig. 1E). No Notch4 transcripts were detected in the homozygotes by either technique. Thus, unlike mouse embryos homozygous for targeted mutations in the Notch1 (Swiatek et al. 1994; Conlon et al. 1995) and Notch2 (Hamada et al. 1999; T. Gridley unpubl.) genes, embryos homozygous for a Notch4 null mutation develop normally. We next examined whether we could detect genetic interactions between the Notch $4^{d 1}$ mutation and a null mutation we had made previously in the Notch1 gene (the Notch $1^{\text {in32 }}$ mutation; Swiatek et al. 1994).

\section{Notch1 $1^{+/-}$Notch4 $4^{-/-}$mutant mice are viable but show growth retardation}

To examine possible genetic interactions between the Notch1 and Notch 4 targeted mutations, we crossed mice heterozygous for the Notch1 $1^{\text {in32 }}$ mutation /designated Notch $1^{+/-} \mid$to mice homozygous for the Notch $4^{d 1}$ mutation $\left(\right.$ Notch $\left.^{-/-}\right)$. Double heterozygous ( Notch $1^{+/-}$ 
A

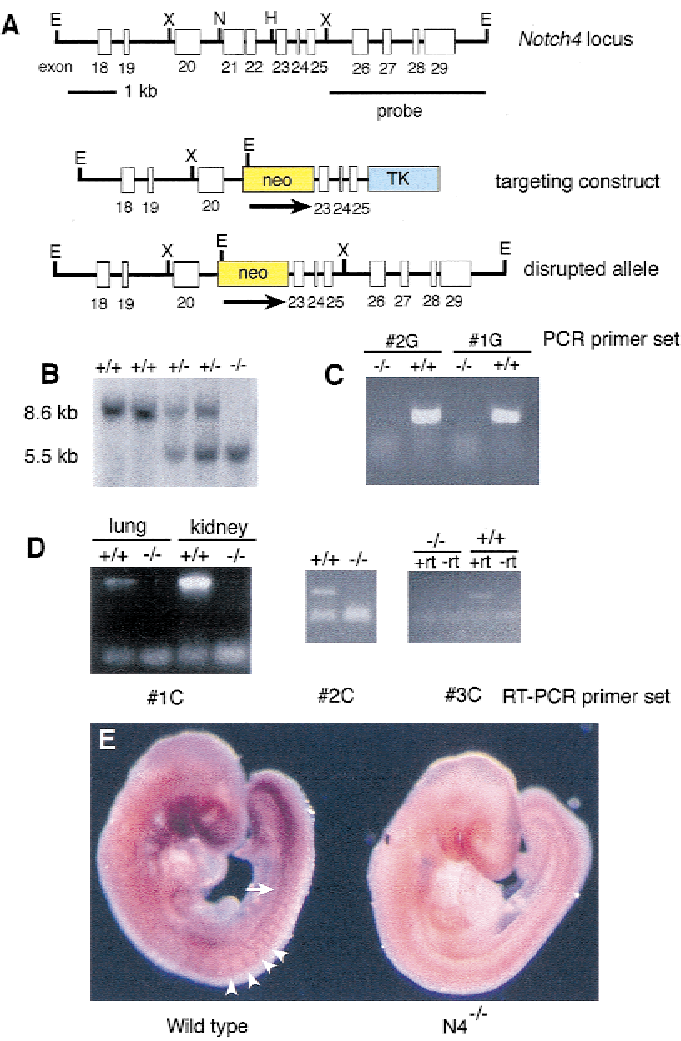

Figure 1. Targeted disruption of the Notch4 gene. (A) Targeting scheme. (Top) The genomic organization of a portion of the Notch 4 gene. Exons are indicated by white boxes. (Middle) The structure of the targeting vector. The deleted exons 21 and 22 encode amino acids 1249-1434 in the extracellular domain of the Notch4 protein. (Bottom) The predicted structure of the Notch4 locus following homologous recombination of the targeting vector. (E) EcoRI; (H) HindIII; (N) NcoI; (X) XbaI. (B) DNA isolated from embryos of the intercross of $\mathrm{Notch}^{+/-}$heterozygous mice was digested with EcoRI, blotted, and hybridized with the indicated probe. Sizes of hybridizing fragments are indicated. Genotypes of progeny are indicated at the top of the lane. $(C)$ Genomic PCR analysis with primers located in region deleted in the Notch $4^{d 1}$ mutant allele. Genotypes are indicated at top. $(D)$ RT-PCR analysis. RT-PCR primer sets are indicated at the bottom of each panel. Primer set \#1C flanks the deleted region; primers sets \#2C and \#3C are located at the 3 ' end of the Notch 4 cDNA. Genotypes are indicated at the top of the lane. $(+r t)$ Plus reverse transcriptase; $(-\mathrm{rt})$ without reverse transcriptase. $(E)$ Whole mount in situ hybridization of a wild-type and a Notch4 ${ }^{-/-}$embryo with an antisense Notch4 riboprobe encoding the intracellular domain of the Notch4 protein. Notch4 expression in the wild-type embryo is observed in intersomitic blood vessels (arrowheads) and the dorsal aorta (arrow). No Notch4 expression is observed in the mutant embryo.

$\mathrm{Notch}^{+/-}$) animals were crossed with $\mathrm{Notch}^{-/-}$mice to generate animals with the genotype Notch1 ${ }^{+/-} \mathrm{Notch}^{4^{-/-}}$. Mice with this genotype appeared smaller than their $\mathrm{Notch}^{+/+} \mathrm{Notch}^{-/-}$littermates. Breeding studies indicated that $\mathrm{Notch1}^{+/-} \mathrm{Notch}^{-/-}$adult mice were fertile. We therefore crossed Notch1 $1^{+-}$Notch4 ${ }^{-/-}$mice with Notch $4^{-/-}$mice and followed the growth rates of the progeny.
This analysis revealed postnatal growth retardation in the Notch1 $^{+/-}$Notch4 $^{-/-}$double mutant animals. At birth, no clear differences in the weight of the pups could be detected. By weaning age (around 3 weeks), however,

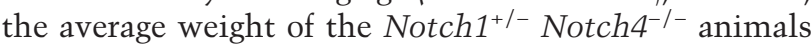
was $~ 80 \%$ that of their $\mathrm{Notch}^{+/+} \mathrm{Notch}^{-/-}$littermates (Fig. 2). Although we have not determined the cause of

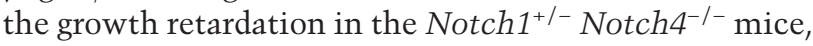
this analysis clearly revealed a genetic interaction between the Notch1 and Notch4 mutations.

\section{Synergistic effects in Notch $1^{-/-}$Notch $4^{-/-}$double homozygous mutant embryos}

We next analyzed whether a genetic interaction between the Notch1 and Notch4 mutations was detectable in double homozygous mutant embryos. Because Notch1 $1^{-/-}$embryos arrest in development at or shortly before E9.5 (Swiatek et al. 1994; Conlon et al. 1995), we isolated embryos at E9.5 from the intercross of Notch1 $^{+/-}$Notch $^{-/-}$mice (Fig. 3). Typically, Notch1 $1^{-/-}$ mutant embryos arrest in development after forming 1620 somites, completing embryonic turning and completing closure of the anterior neural tube (Fig. 3C) (Swiatek et al. 1994; Conlon et al. 1995). Of Notch1 ${ }^{-1-}$ Notch4 $^{-/-}$ double mutant embryos $\sim 50 \%$ were more severely affected than a typical Notch1 $1^{-/}$mutant embryo. These

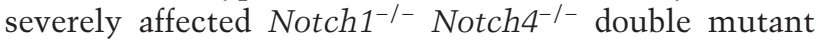
embryos had formed fewer somites, had not completed embryonic turning, and had open neural tubes (Fig. 3A,B). The remaining double mutant embryos appeared phenotypically like Notch1 $1^{-1-}$ mutant embryos. These data indicated that, despite the fact that the Notch4 gene is not essential for embryonic development, the Notch1 and Notch4 genes play partially redundant roles during embryogenesis.

\section{Defects in angiogenic vascular remodeling in $\mathrm{Notch}^{-/-}$} and Notch1 ${ }^{-1-}$ Notch4 ${ }^{-/-}$mutant embryos

Both the Notch1 (Franco del Amo et al. 1992; Reaume et

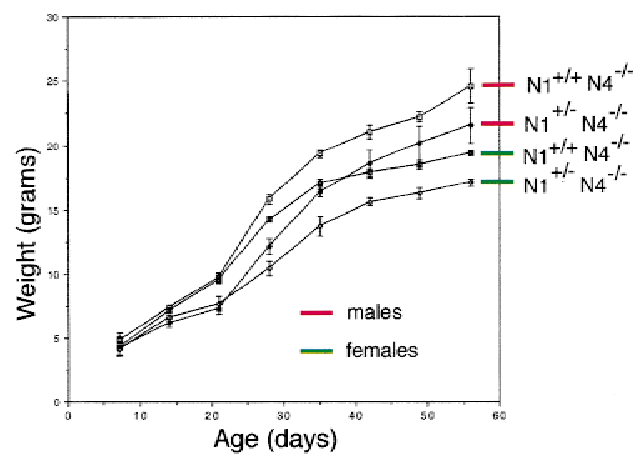

Figure 2. Genetic interactions of Notch1 and Notch4 mutations. Growth curve of Notch1 ${ }^{+/-} \operatorname{Notch}^{-/-}(\bullet, \diamond)$ vs. $\operatorname{Notch}^{+/+} \operatorname{Notch}^{-/-}(\mathbf{\square}, \square)$ littermates. The weights of Notch1 ${ }^{+/-}$Notch4 $^{-/-}$and Notch1 ${ }^{+/+}$Notch4 $^{-/-}$mutant animals are plotted against age. Males (red) and females (green) are plotted separately. Data presented are from four mice in each group. Error bars indicate the S.D.. 

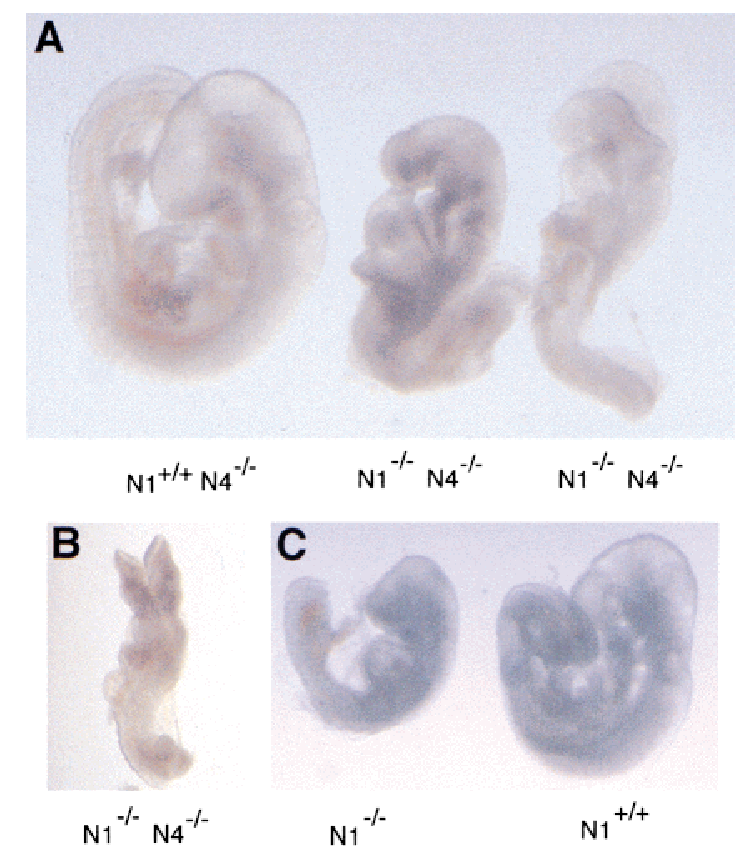

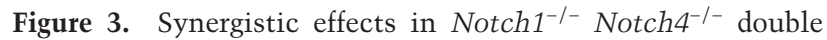
mutant embryos. (A) Two Notch1 ${ }^{-/-}$Notch $^{-/-}$double homozygous mutant embryos and littermate control isolated at E9.5.

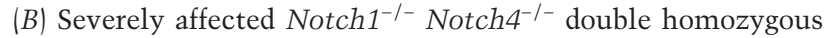
mutant embryo. The double homozygous mutant embryos in both $A$ and $B$ have not completed embryonic turning and have open neural tubes. (C) A typical Notch1 ${ }^{-1-}$ embryo and control littermate at E9.5. The Notch1 $1^{-1-}$ mutant embryo has completed turning and has a closed neural tube.

al. 1992) and Notch4 (Uyttendaele et al. 1996; Shirayoshi et al. 1997; also see Fig. 1E) genes are expressed in vascular endothelial cells, and the Notch1 ${ }^{-/-}$mutant and

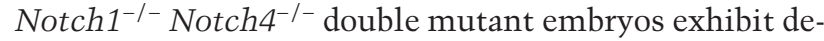
fects (e.g., pericardial edema) suggestive of a failure to maintain vascular homeostasis. We therefore examined

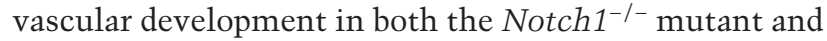
Notch1 $1^{-/}$Notch4 ${ }^{-/-}$double mutant embryos. This analysis revealed substantial defects in angiogenic vascular remodeling in both the Notch1-/- mutant and Notch1 ${ }^{-/-}$Notch4 ${ }^{-/-}$double mutant embryos.

Vascular defects can first be observed in the yolk sacs of the mutant embryos. When viewed in whole mount at E9.5, yolk sacs from wild type (not shown), Notch1 ${ }^{+/-}$ (Fig. 4A), and Notch4 ${ }^{-1-}$ (Fig. 4B) embryos all exhibited large vitelline blood vessels. All Notch1-/- mutant and

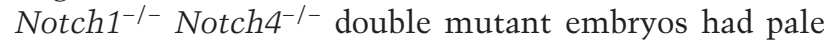
yolk sacs lacking obvious blood vessels (Fig. 4C,D). We then visualized the vascular network of mutant embryos and littermate controls by staining with a monoclonal antibody to platelet endothelial cell adhesion molecule-1 (PECAM-1), a specific marker for vascular endothelial cells (Baldwin et al. 1994). In the yolk sacs of both

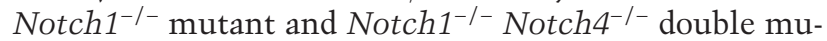
tant embryos, the primary vascular plexus appeared to form normally, indicating that there were no apparent defects in vasculogenesis in the mutants. However, the mutant embryos failed to remodel the primary vascular plexus to form the large and small blood vessels of the mature yolk sac (Fig. 4E-G). Histological analysis of PECAM-1-stained yolk sacs demonstrated that both small capillaries and large vitelline collecting vessels differentiated in control yolk sacs (Fig. 4H), whereas both Notch1 ${ }^{-/-}$(Fig. 4I) and Notch1 ${ }^{-/-}$Notch4 $^{-/-}$(not shown) yolk sacs exhibited a disorganized, confluent vascular plexus.

Vascular defects were also observed in the labyrinthine layer of the placentas of the mutant embryos (Fig. 4J, K; data not shown). In both Notch1-/- mutant and Notch1-/- Notch4 ${ }^{-/-}$double mutant embryos at E9.5,
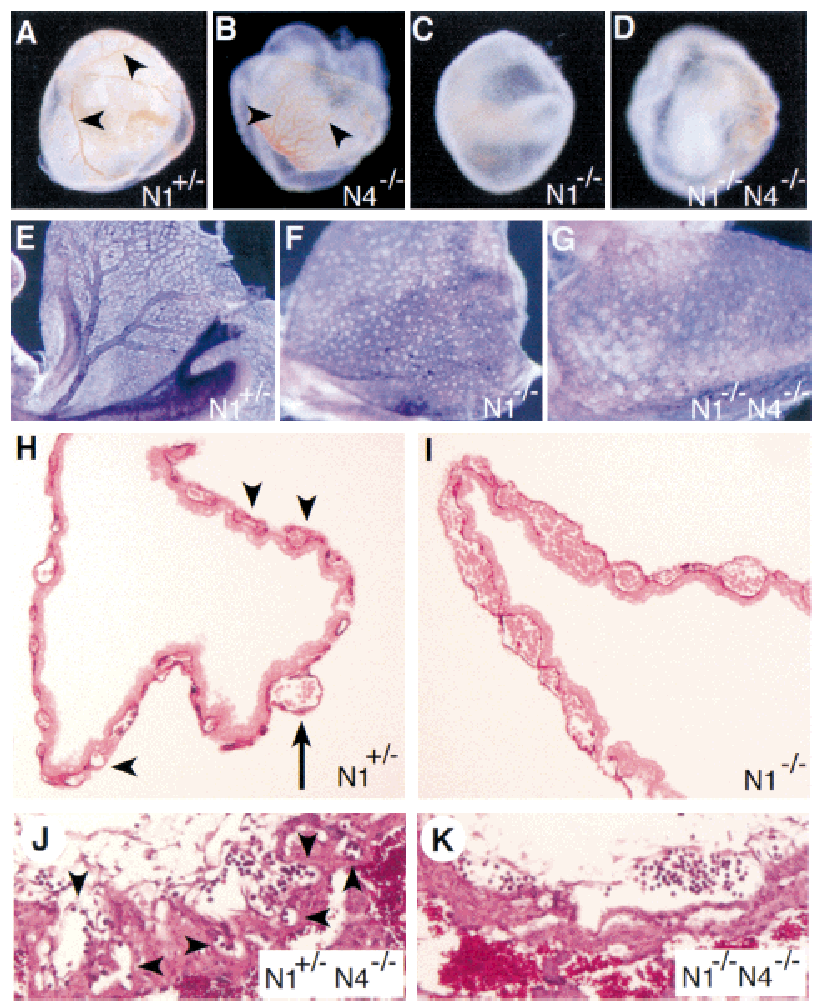

Figure 4. Yolk sac and placental defects in mutant embryos. $(A-D)$ Morphology of embryos in their yolk sacs at E9.5. Large vitelline blood vessels (arrowheads) are observed in the Notch $^{+/-}$and Notch4 ${ }^{-/-}$yolk sacs, but not in the Notch1-/and Notch1 ${ }^{-/-}$Notch4 $^{-/-}$mutants. (E-G) PECAM-1-stained

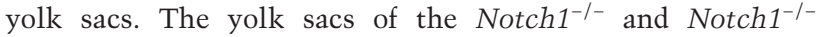
Notch $4^{-/-}$mutant embryos are at the primitive vascular plexus stage and have not undergone vascular remodeling to form the large and small blood vessels of the mature yolk sac. $(H, I)$ Histological sections of PECAM-1-stained yolk sacs. The Notch1 ${ }^{+/-}$ yolk sac $(H)$ has differentiated both small capillaries (arrowheads) and large vitelline collecting vessels (arrow). The Notch1 ${ }^{-1-}$ yolk sac $(I)$ exhibits a disorganized, confluent vascular plexus. $(J, K)$ Histological sections of placentas at E9.5. In the Notch $1^{+/-}$Notch $^{-/-}$control embryo $(J)$, embryonic blood vessels containing nucleated erythrocytes (arrowheads) have invaded the labyrinthine layer of the placenta. In the Notch1-/Notch $4^{-1-}$ mutant embryo $(K)$, embryonic blood vessels are present at the edge of the placenta but have not invaded the labyrinthine layer. 
the allantois of the mutant embryos fused with the chorionic plate, which is an initiating step in the formation of a functional chorioallantoic placenta. However, in the control embryos (e.g., the Notch $1^{+/-}$Notch $4^{-/-}$embryo shown in Fig. 4J, which is phenotypically wild type), blood vessels containing embryonic nucleated erythrocytes could be observed invading and interdigitating into the labyrinthine trophoblast layer of the developing pla-

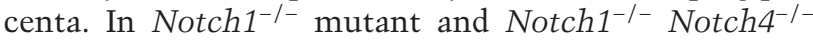
double mutant embryos, embryonic blood vessels containing nucleated erythrocytes remained at the periphery of the trophoblast layer and did not invade the labyrinthine layer (Fig. 4K). The failure of mutant endothelial cells to efficiently invade the labyrinthine region provides further evidence of defects in angiogenesis in the

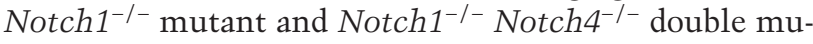
tant embryos.

In addition to the vascular defects observed in the yolk sac and placenta, extensive defects in vascular morphogenesis were observed in the mutant embryos themselves (Fig. 5). A prominent feature in the Notch1 ${ }^{-/-}$mutant and Notch $1^{-/-}$Notch $4^{-/-}$double mutant embryos was malformation of the large blood vessels in the anterior of the embryo. The primary vessels for arterial flow to the head of the mammalian embryo are the dorsal aortae, and the primary vessels for venous return are the anterior cardinal veins. In the Notch4 ${ }^{-/-}$(phenotypically wild type) PECAM-1-stained embryo shown in Figure $5 \mathrm{~A}$, the anterior cardinal vein (arrowhead) was easily visualized as a large vessel running in an anterior-posterior direction just beneath the surface ectoderm of the embryo. In the Notch1 $1^{-1-}$ embryo shown in Figure 5B, the anterior cardinal vein (arrowhead) had formed, but was smaller than in the control. Two Notch1 ${ }^{-/-}$Notch $^{-/-}$ double mutant embryos at E9.5 are shown in Figure 5, C and D. In the less severely affected double mutant embryo, discontinuous remnants of the anterior cardinal vein were observed (arrowheads in Fig. 5C). In the more

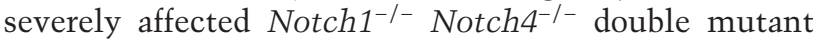
embryo, the anterior cardinal vein appeared to be entirely missing (Fig. 5D). In the trunk of wild-type embryos at E9.5, intersomitic blood vessels were apparent along the boundaries between adjacent somites (Fig. 5E). On the dorsal side of the embryo, the intersomitic vessels branch to form a highly anastomosed capillary net-

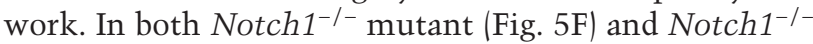
Notch4 ${ }^{-/}$double mutant (Fig. 5G) embryos, intersomitic vessels were severely disorganized.

Histological analyses of PECAM-1-stained embryos confirmed that dorsal aortae and anterior cardinal veins were frequently atretic in the mutant embryos (Fig. 5HJ). In the section of the Notch $1^{+/-}$(phenotypically wild type) embryo shown in Figure $5 \mathrm{H}$, both the dorsal aortae and the anterior cardinal veins had open lumens that were lined with a continuous layer of PECAM-1-positive endothelial cells. In the Notch $1^{-/-}$embryo shown in Figure 5I, the anterior cardinal veins appeared relatively normal, but both dorsal aortae had a collapsed appearance. In the more severely affected Notch1 ${ }^{-1-}$ Notch $^{-1-}$ double mutant embryo (Fig. 5J), endothelial cells were
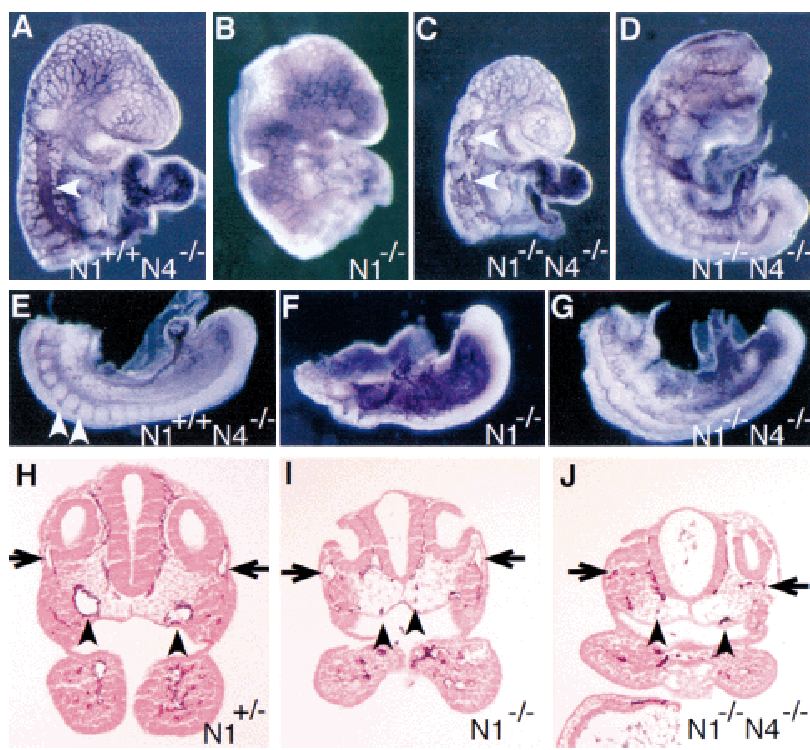

I

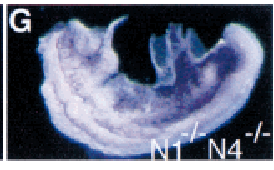

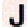

Figure 5. Defects in vascular remodeling in Notch1-/- and

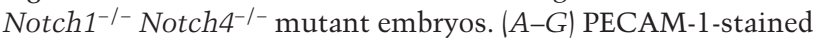
whole mount embryos. $(A-D)$ Defective morphogenesis of the main trunk of the anterior cardinal vein (arrowhead) in Notch1 ${ }^{-/-}(B)$ and Notch $1^{-/-}$Notch $^{-/-}$mutant embryos $(C, D)$. The double mutant embryo in $D$ is more severely affected than the embryo in C. (E-G) In the Notch1 ${ }^{+/-}$Notch $^{-/-}$control embryo $(E)$, intersomitic vessels (arrowheads) differentiate,

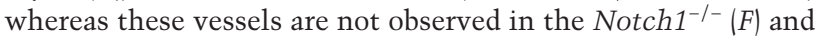

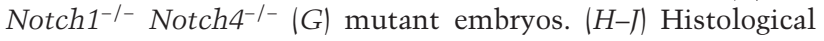
sections of PECAM-1-stained embryos at the level of the otic vesicle. In the $N 1^{+/-}$control embryo $(H)$, both the dorsal aortae (arrowheads) and the anterior cardinal veins (arrows) have open

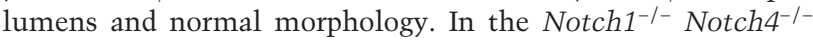
mutant embryo $(J)$, endothelial cells have differentiated but both the dorsal aortae and the anterior cardinal veins have an abnormal, collapsed morphology. In the less-severely affected Notch $1^{-/-}$mutant embryo $(I)$, the anterior cardinal veins still have an open lumen but the dorsal aortae are collapsed.

present at the correct positions of the anterior cardinal veins and dorsal aortae, but no vessel lumens were apparent.

The Dll4 gene encodes the probable ligand for the Notch1 and Notch4 receptors in the early embryonic vasculature

Previous work has shown that both Jag1 and Dll1 homozygous mutant embryos die from vascular defects and hemorrhaging (Xue et al. 1999; Hrabé de Angelis et al. 1997). However, in both these cases, embryonic lethality does not occur until E10.5, at least a day later than in

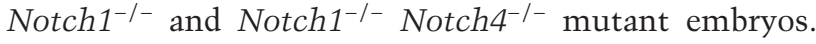
We therefore examined the RNA expression pattern of all the genes encoding Notch ligands at E8.5 and E9.5 (Fig. 6; data not shown). Only the D114 gene (Shutter et al. 2000) was expressed in a spatial and temporal pattern that suggested it could encode the ligand for the Notch1 and Notch4 receptors during early vascular development. At E8.5, D114 expression was observed in the dor- 

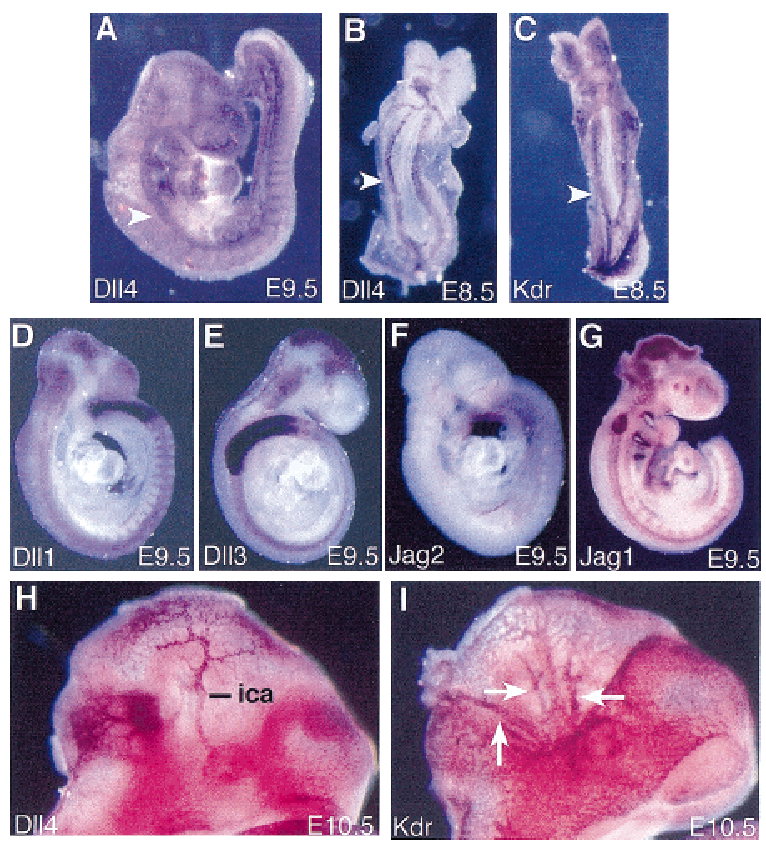

Figure 6. Expression pattern of the Dl14 gene suggests that it encodes the ligand for the Notch1 and Notch4 receptors in the embryonic vasculature. Whole-mount in situ hybridization with the indicated probes of embryos isolated at E8.5 $(B, C), \mathrm{E} 9.5$ $(A, D-G)$, and E10.5 $(H, I)$. D114 expression is observed in the dorsal aorta (arrowheads) and, at E9.5, the intersomitic vessels $(A)$. The other genes encoding Notch ligands do not exhibit obvious expression in the vasculature at E9.5 (D-G). At E10.5, Dl14 expression is observed in the internal carotid artery (ica) $(H)$, but is not observed in branches of the primary head vein (arrows in $I$ ), which express $K d r$.

sal aortae and the endocardium (Fig. 6B). Comparision of Dll4 expression with Kdr (formerly Flk1) expression (Fig. 6C), which is expressed in all endothelial cells and their precursors (Yamaguchi et al. 1993), indicated that Dll4 was expressed in only a subset of endothelial cells. Analysis of D114 expression at E9.5 and E10.5 (Fig. 6A,H) demonstrated that D114 was preferentially expressed in endothelial cells of the arterial system. In contrast, none of the other genes encoding Notch ligands exhibited obvious expression in the vascular system at E8.5 or E9.5 (Fig. 6D-G; data not shown), indicating that these ligands most likely are not involved in activation of the Notch 1 and Notch4 receptors at these stages. We also

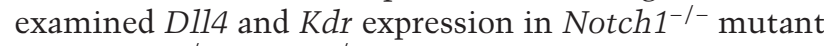
and Notch1 ${ }^{-1-}$ Notch $^{-/-}$double mutant embryos. Dll4 expression was not down-regulated in the mutants, but the expression patterns of both Dll4 and Kdr revealed disruptions in vascular morphogenesis similar to those revealed by PECAM-1 staining of mutant embryos (Fig. 7).

\section{Discussion}

The Notch4 gene is not essential, but the Notch4 mutation genetically interacts with a Notch1 mutation

To determine the biological role of the Notch4 gene in mice, we constructed and analyzed animals homozygous for a deletion that removed exons 21 and 22, which encode 186 amino acids of the extracellular domain of the Notch4 protein. The two closest remaining exons, exon 20 and exon 23, are out of frame with respect to each other. Therefore, a partially functional Notch 4 protein that contains the intracellular (i.e., signal-transducing) domain of the Notch4 protein could not be produced by alternative splicing from exon 20 to exon 23. We also used RT-PCR and in situ hybridization analyses to assay for alternatively spliced Notch4 transcripts that would contain the region of the Notch4 cDNA encoding the intracellular domain of the Notch4 protein. We found no

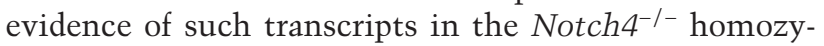
gous mutant mice. These results suggest that the Notch $4^{d 1}$ allele causes a null mutation in the Notch4 gene. Our results reveal that the Notch4 gene is dispensable for apparently normal embryonic growth in mice, and that $\mathrm{Notch}^{-/-}$adults are viable and fertile. In contrast, both the Notch1 (Swiatek et al. 1994; Conlon et al. 1995) and Notch2 (Hamada et al. 1999; T. Gridley, unpubl.) genes are essential for embryonic development. It should be noted that all Notch ligands for which either spontaneous or targeted mutations have been isolated are also essential for proper embryonic development in mice. This includes mutations in the Jag1 (Xue et al. 1999), Jag2 (Sidow et al. 1997; Jiang et al. 1998), Dl11 (Hrabé de Angelis et al. 1997), and Dll3 (Kusumi et al. 1998) genes.

The Notch 4 gene is primarily expressed in vascular endothelial cells during early embryogenesis in mice (Uyttendaele et al. 1996; Shirayoshi et al. 1997). Because the Notch1 gene is expressed in endothelial cells at the same stages as the Notch4 gene (Franco del Amo et al.
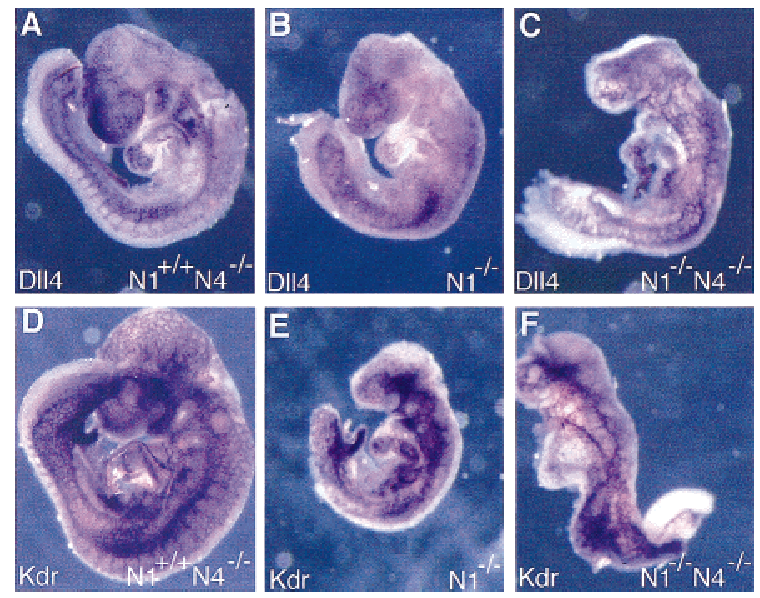

Figure 7. D114 and $K d r$ expression in mutant embryos. Embryos of the indicated genotypes were hybridized with riboprobes for $D 114(A-C)$ and $K d r(D-F)$. Note the more severe dys-

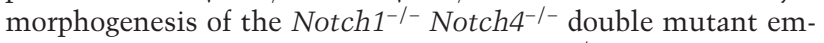
bryos $(C, F)$ compared with the Notch1 ${ }^{-/-}$single mutant embryos $(B, E)$. $D 114$ expression was not downregulated in either

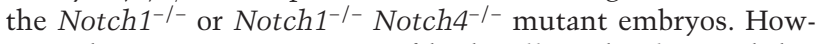
ever, the expression patterns of both D114 and $K d r$ reveal disruptions in vascular morphogenesis in the mutant embryos. 
1992; Reaume et al. 1992), we generated Notch1/Notch4 double mutant mice and embryos to assess whether there were genetic interactions between these two mutations. These experiments revealed genetic interactions between the Notch1 and Notch 4 mutations at two different stages. Adult Notch $1^{+/-}$Notch $4^{-/-}$double mutant mice were viable and fertile, but exhibited postnatal growth deficiency. Although Notch1 ${ }^{+/-}$Notch4 $^{-/-}$ double mutant mice were the same size as their Notch $1^{+/+}$Notch4 ${ }^{-/-}$littermates at birth, their postnatal growth deficiency quickly became apparent. This was clearly seen at weaning age, when the weights of Notch1 $1^{+/-} \mathrm{Notch}^{-/-}$double mutant mice averaged $\sim 80 \%$ that of their littermates.

We also observed genetic interactions between the Notch4 and Notch1 mutations during embryonic devel-

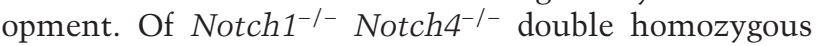
mutant embryos $\sim 50 \%$ had a more severe phenotype than Notch $1^{-/-}$mutant embryos. These severely affected Notch1 $1^{-/}$Notch4 ${ }^{-/-}$double mutant embryos formed fewer somites than Notch1-1- mutant embryos and did not complete embryonic turning or neural tube closure. Vascular defects were frequently more severe in the Notch1 $1^{-/}$Notch4 $4^{-/-}$double mutant embryos than in Notch $1^{-/-}$single mutant embryos (e.g., see Fig. 5). We hypothesize that the more severe phenotype of the

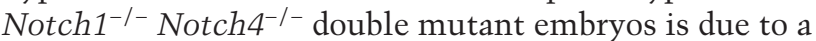
more severely compromised vascular system than in Notch $1^{-/-}$mutant embryos, thereby leading to more severe growth retardation in the double mutant embryos. This interaction is not completely penetrant, as only

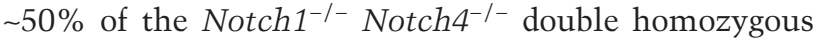
mutant embryos exhibit the more severe phenotype. We do not know the cause of this incomplete penetrance. However, it may be due to the segregation of modifier loci in the mixed C57BL/6J $\times 129 /$ SvImJ genetic background on which the mutant alleles are maintained.

\section{Notch signaling is required for angiogenic vascular remodeling}

Targeted mutations in components of a variety of signaling pathways (e.g., vascular endothelial growth factors, TGF- $\beta 1$, angiopoietins, ephrins) have been shown to regulate vascular morphogenesis in mice (for review, see Folkman and D'Amore 1996; Hanahan 1997; Carmeliet and Collen 1999; Gale and Yancopoulos 1999; Neufeld et al. 1999). Our results demonstrate that another major intercellular signaling pathway, the Notch pathway, also regulates vascular morphogenesis and angiogenic vascular remodeling. We observed vascular defects in the placenta, yolk sac, and embryo proper of both Notch1-/-

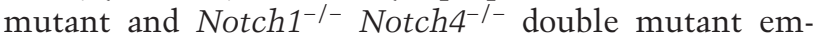
bryos. In the yolk sac of the mutant embryos, the primary vascular plexus formed normally, indicating that there were no apparent defects in vasculogenesis in the mutants. However, both Notch1 ${ }^{-1-}$ mutant and Notch1 ${ }^{-/-}$Notch4 $4^{-/-}$double mutant embryos failed to remodel the primary vascular plexus to form the large vitelline blood vessels, a process that occurs by angio- genesis. Defects in angiogenesis were also apparent in the placenta, where embryonic blood vessels failed to invade the placental labyrinth. In the embryo proper, defects in angiogenesis and vascular remodeling were apparent as malformations of major vessels such as the dorsal aortae, the anterior cardinal veins, and the intersomitic blood vessels.

Our expression analysis of the genes encoding ligands for Notch-family receptors indicated that only the newly cloned Dll4 gene (Shutter et al. 2000) was expressed in the embryonic vasculature early enough to encode the ligand responsible for activation of the Notch1 and Notch4 receptors. The preferential expression of D114 in endothelial cells of the arterial system is strongly reminiscent of the differential expression of ephrin ligands and their Eph receptors in the arterial and venous systems (Wang et al. 1998; Adams et al. 1999; Gerety et al. 1999; for review, see Gale and Yancopoulos 1999). During early vascular development, the ephrinB2 gene is expressed in arterial endothelium, whereas its receptor EphB4 is expressed in venous endothelium. Embryos homozygous for targeted mutations of ephrinB2 (Wang et al. 1998; Adams et al. 1999) and EphB4 (Gerety et al. 1999) exhibit defects in angiogenesis and vascular remodeling that are essentially identical to the vascular defects we observe in the Notch1 $1^{-/-}$mutant and

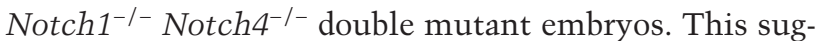
gests the intriguing possibility that the Notch pathway and the ephrin/Eph pathway functionally interact during the intercellular signaling events that must be involved in remodeling the embryonic vasculature. Further work, including genetic epistasis experiments with various Notch pathway and ephrin/Eph pathway mutants, will be required to distinguish whether these two contactmediated intercellular signaling pathways interact, or whether they function independently, during vascular development and remodeling.

A role for the Notch pathway in vascular homeostasis in adult humans has been indicated by the finding that the degenerative vascular disease CADASIL is caused by missense mutations in the Notch3 gene (Joutel et al. 1996). Our studies reveal a previously unappreciated role for the Notch signaling pathway in regulating angiogenic vascular remodeling during embryonic development in mice. Taken together, these results demonstrate that the Notch signaling pathway is essential both for vascular development in embryos and maintenance of vascular homeostasis in adults.

\section{Partial functional redundancy between the Notch1 and Notch4 genes}

Notch genes form a multigene family in vertebrates, and our results provide the first evidence for functional redundancy in the Notch signaling pathway in mammals. Previous experiments in Caenorhabditis elegans had revealed functional redundancy in the Notch signaling pathway. Although only a single Notch gene is known in Drosophila, C. elegans contains two genes encoding Notch-family receptors, 1 in-12 and $g l p-1$. Lambie and 
Kimble (1991) showed that the 7 in-12 and glp-1 genes were functionally redundant, as double homozygous mutant embryos exhibit a novel, and more severe, synergistic phenotype. The redundancy of $g l p-1$ and lin-12 gene action was further supported by the finding that expression of $g l p-1$ coding sequences under control of lin-12 regulatory sequences can rescue the lin-12 null phenotype (Fitzgerald et al. 1993), and by the ability of a particular neomorphic $g l p-1$ allele to mimic dominant gain of function lin-12 mutations (Mango et al. 1991). These results suggested that the Glp-1 and Lin-12 proteins are biochemically interchangeable, and that their distinct roles during development are due largely to differences in gene expression. Our results extend these findings to mammals and demonstrate that although the Notch4 gene plays no essential role in laboratory mice, the Notch1 and Notch4 genes have partially overlapping roles during embryogenesis.

\section{Materials and methods}

\section{Targeting vector construction}

A Notch4 genomic clone (Gallahan and Callahan 1997) isolated from a $\mathrm{P}_{1}$ library was used for construction of the targeting vector. The sequence of the mouse Notch 4 gene is available in GenBank (accession no. AF030001). For construction of the targeting vector, 3.3-kb EcoRI-NcoI fragment of the Notch4 gene was subcloned upstream of a PGK-neo expression cassette (Soriano et al. 1991), and a 1.1-kb HpaI-XbaI Notch4 fragment was subcloned downstream of the PGK-neo cassette. This resulted in the deletion of a 1.0-kb Notch4 genomic fragment containing exons 21 and 22 of the Notch4 gene. We refer to this allele as Notch $4^{d 1}$. The official nomenclature for the alleles used in these studies are Notch $4^{d 1}$ : Notch4 ${ }^{\text {tm1Grid }}$; Notch1 ${ }^{\text {in32 }}$ (Swiatek et al. 1994): Notch1 $1^{\text {tm1Grid }}$. A HSV-tk cassette was introduced to allow negative selection against random integration of the targeting vector (Mansour et al. 1988).

Electroporation, selection, and screening of ES cells and mouse genotyping

CJ7 ES cells were electroporated with $25 \mu \mathrm{g}$ of linearized targeting vector, selected, screened, and injected into blastocysts from C57BL/6J mice as described previously (Swiatek and Gridley 1993). For Southern blot analysis, a 2.4-kb XbaI-EcoRI fragment was used as probe. Germ-line transmission was obtained for two independently targeted ES cell clones. Animals were genotyped by PCR or by Southern blot analysis. PCR primers for the wildtype Notch4 allele were (5'-CCTATGACCAGTACTGCCGAG-3') and (5'-GAGGAATCCCTAGCTCCACTC-3'), yielding an amplification product of $312 \mathrm{bp}$; the primers for the Notch $4^{d 1}$ allele were (5'-GAGGAGTGTCTCTTTGATGGC-3') and (5'-AAGCGCATGCTCCAGACTGCC-3'), yielding an amplification product of $\sim 500 \mathrm{bp}$.

For confirmation that the expected fragment of the Notch4 gene was deleted in the Notch $4^{d 1}$ homozygous mutant mice, genomic DNA was isolated from Notch4 ${ }^{d 1}$ adult homozygotes and wild-type controls and analyzed with PCR primers from the deleted region. Two primer sets were used: \#1G forward $\left(5^{\prime}\right.$ CTTTCCCTTCCCACAGGTTTG-3') and \#1G reverse (5'TTGGTTCTATCTCTCCCAGCGAGG-3'), which amplify a 257-bp product; \#2G forward (5'-AGGTTTGTGGTAGTGATG-
GGAGTG-3') and \#2G reverse (5'-ACTGAATAGATGGGACAGGGGG-3'), which amplify a 322 -bp product.

\section{RT-PCR analysis}

Total RNA was isolated from kidney and lung tissues of adult homozygous Notch $4^{d 1}$ mutants and wild-type littermates. For the RT-PCR analysis, cDNA was synthesized with oligo(dT) primer, and Notch4 cDNAs were detected by PCR. Two RTPCR primer sets were used. One primer set was chosen to flank the region deleted in the Notch $4^{d 1}$ mutant allele: \#1C forward (5'-ACCCTGCTCCAATGGAGGAT-3', encoding amino acids 1049-1055) and \#1C reverse (5'-AGAACCTCCGATTCACACTCC-3', encoding amino acids 1564-1570), generating an amplification product of $1.3 \mathrm{~kb}$ (Smith et al. 1995).

Additional primer sets were chosen to amplify the $3^{\prime}$ end of any potential alternatively spliced transcripts from the Notch $4^{d 1}$ mutant allele: \#2C forward (5'-TGGAGCGGATAAAGATGCCC-3', encoding amino acids $1752-1758$ ) and \#2C reverse (5'-AGCGTTAGCAGGTCCCAGTGAC-3'), encoding amino acids 1806-1813), generating an amplification product of 185 base pairs; \#3C forward (5'-CCAAGAGATTCCCTTAAACTCGG-3', encoding amino acids 1952-1958) and \#3C reverse (5'-CCAGAGTTTAGGGATTCTCG-3', located in the $3^{\prime}$ untranslated region), generating an amplification product of $260 \mathrm{bp}$. Because both primer \#3C forward and \#3C reverse are located in exon 29, the large 3' terminal exon of the Notch4 gene (Gallahan and Callahan 1997), total RNA was treated with ribonuclease-free DNase prior to synthesis of cDNA with oligo(dT) primer.

Histology, in situ hybridization, and immunohistochemistry

Organs from adult mice were fixed by immersion in Fekete's acid-alcohol-formalin and were processed as described (Relyea et al. 1999). Embryos were dissected and DNA was prepared from the yolk sacs or tails for genotyping by PCR or by Southern blot analysis. Embryos for whole-mount in situ hybridization were fixed in $4 \%$ paraformaldehyde in phosphate-buffered saline (PBS) overnight at $4^{\circ} \mathrm{C}$. Embryos for immunohistochemistry were fixed in $4 \%$ paraformaldehyde in PBS and stained with a monoclonal anti-PECAM-1 antibody (clone MEC 13.3; Pharmingen).

\section{Acknowledgments}

We thank J. Barker, T. O'Brien, B. McCright, and E. Carver for comments on the manuscript. This work was supported by grants to T.G. from the NIH (NS36437) and the March of Dimes Birth Defects Foundation (FY99-290), and to J.K. from the NIH (HL62454). L.T.K. was supported by a NRSA postdoctoral fellowship from NHLBI and a Training Grant from NICHD to The Jackson Laboratory. This work was also supported by a grant (CA34196) from the National Cancer Institute to The Jackson Laboratory.

The publication costs of this article were defrayed in part by payment of page charges. This article must therefore be hereby marked "advertisement" in accordance with 18 USC section 1734 solely to indicate this fact.

\section{References}

Adams, R.H., G.A. Wilkinson, C. Weiss, F. Diella, N.W. Gale, U. Deutsch, W. Risau, and R. Klein. 1999. Roles of ephrinB ligands and EphB receptors in cardiovascular development: 
Demarcation of arterial/venous domains, vascular morphogenesis, and sprouting angiogenesis. Genes \& Dev. 13: 295306.

Artavanis-Tsakonas, S., M.D. Rand, and R.J. Lake. 1999. Notch signaling: Cell fate control and signal integration in development. Science 284: 770-776.

Baldwin, H.S., H.M. Shen, H.C. Yan, H.M. De Lisser, A. Chung, C. Mickanin, T. Trask, N.E. Kirschbaum, P.J. Newman, S.M. Albeda, and C.A. Buck. 1994. Platelet endothelial cell adhesion molecule-1 (PECAM-1/CD31): Alternatively spliced, functionally distinct isoforms expressed during mammalian cardiovascular development. Development 120: 2539-2553.

Carmeliet, P. and D. Collen. 1999. Role of vascular endothelial growth factor and vascular endothelial growth factor receptors in vascular development. Curr. Top. Microbiol. Immunol. 237: 133-158.

Chan, Y.-M. and Y.N. Jan. 1998. Roles for proteolysis and trafficking in Notch maturation and signal transduction. Cell 94: 423-426.

Conlon, R.A., A.G. Reaume, and J. Rossant. 1995. Notch1 is required for the coordinate segmentation of somites. Development 121: 1533-1545.

Fitzgerald, K., H.A. Wilkinson, and I. Greenwald. 1993. glp-1 can substitute for lin-12 in specifying cell fate decisions in Caenorhabditis elegans. Development 119: 1019-1027.

Folkman, J. and P.A. D'Amore. 1996. Blood vessel formation: What is its molecular basis? Cell 87: 1153-1155.

Franco del Amo, F., D.E. Smith, P.J. Swiatek, M. GendronMaguire, R.J. Greenspan, A.P. McMahon, and T. Gridley. 1992. Expression of Motch, a mouse homolog of Drosophila Notch, suggests an important role in early postimplantation mouse development. Development 115: 737-745.

Gale, N.W. and G.D. Yancopoulos. 1999. Growth factors acting via endothelial cell-specific receptor tyrosine kinases: VEGFs, angiopoietins, and ephrins in vascular development. Genes \& Dev. 13: 1055-1066.

Gallahan, D. and R. Callahan. 1987. Mammary tumorigenesis in feral mice: Identification of a new int locus in mouse mammary tumor virus (Czech II)-induced mammary tumors. J. Virol. 61: 66-74.

- 1997. The mouse mammary tumor associated gene INT3 is a unique member of the NOTCH gene family (NOTCH4). Oncogene 14: 1883-1890.

Gerety, S.S., H.U. Wang, Z.F. Chen, and D.J. Anderson. 1999. Symmetrical mutant phenotypes of the receptor EphB4 and its specific transmembrane ligand ephrin-B2 in cardiovascular development. Mol. Cell 4: 403-414.

Greenwald, I. 1998. LIN-12/Notch signaling: Lessons from worms and flies. Genes \& Dev. 12: 1751-1762.

Gridley, T. 1997. Notch signaling in vertebrate development and disease. Mol. Cell. Neurosci. 9: 103-108.

Hamada, Y., Y. Kadokawa, M. Okabe, M. Ikawa, J.R. Coleman, and Y. Tsujimoto. 1999. Mutation in ankyrin repeats of the mouse Notch2 gene induces early embryonic lethality. Development 126: 3415-3424.

Hanahan, D. 1997. Signaling vascular morphogenesis and maintenance. Science 277: 48-50.

Hrabé de Angelis, M., J. McIntyre II, and A. Gossler. 1997. Maintenance of somite borders in mice requires the Delta homologue Dll1. Nature 386: 717-721.

Jiang, R., Y. Lan, H.D. Chapman, C. Shawber, C.R. Norton, D.V. Serreze, G. Weinmaster, and T. Gridley. 1998. Defects in limb, craniofacial and thymic development in Jagged2 mutant mice. Genes \& Dev. 12: 1046-1057.

Joutel, A., C. Corpechot, A. Ducros, K. Vahedi, H. Chabriat, P. Mouton, S. Alamowitch, V. Domenga, M. Cécillion, E.
Maréchal et al. 1996. Notch3 mutations in CADASIL, a hereditary adult-onset condition causing stroke and dementia. Nature 383: 707-710.

Kusumi, K., E. Sun, A.W. Kerrebrock, R.T. Bronson, D.-C. Chi, M.S. Bulotsky, J.B. Spencer, B.W. Birren, W.N. Frankel, and E.S. Lander. 1998. The mouse pudgy mutation disrupts Delta homologue DI13 and initiation of early somite boundaries. Nat. Genet. 19: 274-278.

Lambie, E.J. and J. Kimble. 1991. Two homologous regulatory genes, lin-12 and glp-1, have overlapping functions. Development 112: 231-240.

Mango, S.E., E.M. Maine, and J. Kimble. 1991. Carboxy-terminal truncation activates glp-1 protein to specify vulval fates in Caenorhabditis elegans. Nature 352: 811-815.

Mansour, S.L., K.R. Thomas, and M.R. Capecchi. 1988. Disruption of the proto-oncogene int-2 in mouse embryo-derived stem cells: A general strategy for targeting mutations to nonselectable genes. Nature 336: 348-352.

Neufeld, G., T. Cohen, S. Gengrinovitch, and Z. Poltorak. 1999. Vascular endothelial growth factor (VEGF) and its receptors. FASEB J. 13: 9-22.

Reaume, A.G., R.A. Conlon, R. Zirngibl, T.P. Yamaguchi, and J. Rossant. 1992. Expression analysis of a Notch homologue in the mouse embryo. Dev. Biol. 154: 377-387.

Relyea, M.J., J. Miller, D. Boggess, and J.P. Sundberg. 1999. Necropsy methods for laboratory mice. Biological characterization of a new mutation. In Systematic approach to evaluation of mouse mutations (ed. J.P. Sundberg and D. Boggess), pp. 57-89. CRC Press, Inc., Boca Raton, FL.

Risau, W. 1997. Mechanisms of angiogenesis. Nature 386: 671674.

Risau, W. and I. Flamme. 1995. Vasculogenesis. Annu. Rev. Cell Dev. Biol. 11: 73-91.

Robbins, J., B.J. Blondel, D. Gallahan, and R. Callahan. 1992. Mouse mammary tumor gene int-3: A member of the notch gene family transforms mammary epithelial cells. J. Virol. 66: 2594-2599.

Shirayoshi, Y., Y. Yuasa, T. Suzuki, K. Sugaya, E. Kawase, T. Ikemura, and N. Nakatsuji. 1997. Proto-oncogene of int-3, a mouse Notch homologue, is expressed in endothelial cells during early embryogenesis. Genes \& Cells 2: 213-224.

Shutter, J.R., S. Scully, G. Deblandre, C.R. Kintner, and K.L. Stark. 2000. Delta4, a novel Notch ligand expressed in arterial endothelium. Genes \& Dev. (this issue).

Sidow, A., M.S. Bulotsky, A.W. Kerrebrock, R.T. Bronson, M.J. Daly, M.P. Reeve, T.L. Hawkins, B.W. Birren, R. Jaenisch, and E.S. Lander. 1997. Serrate2 is disrupted in the mouse limb-development mutant syndactylism. Nature 389: 722 725.

Smith, G.H., D. Gallahan, F. Diella, C. Jhappan, G. Merlino, and R. Callahan. 1995. Constitutive expression of a truncated INT3 gene in mouse mammary epithelium impairs differentiation and functional development. Cell Growth Differ. 6: $563-577$.

Soriano, P., C. Montgomery, R. Geske, and A. Bradley. 1991. Targeted disruption of the c-src proto-oncogene leads to osteopetrosis in mice. Cell 64: 693-702.

Swiatek, P. and T. Gridley. 1993. Perinatal lethality and defects in hindbrain development in mice homozygous for a targeted mutation of the zinc finger gene Krox20. Genes \& Dev. 7: 2071-2084.

Swiatek, P.J., C.E. Lindsell, F. Franco del Amo, G. Weinmaster, and T. Gridley. 1994. Notch1 is essential for postimplantation development in mice. Genes \& Dev. 8: 707-719.

Uyttendaele, H., G. Marazzi, G. Wu, Q. Yan, D. Sassoon, and J. Kitajewski. 1996. Notch4/int-3, a mammary proto-onco- 
Krebs et al.

gene, is an endothelial cell-specific mammalian Notch gene. Development 122: 2251-2259.

Uyttendaele, H., J.V. Soriano, R. Montesano, and J. Kitajewski. 1998. Notch4 and Wnt-1 proteins function to regulate branching morphogenesis of mammary epithelial cells in an opposing fashion. Dev. Biol. 196: 204-217.

Wang, H.U., Z.F. Chen, and D.J. Anderson. 1998. Molecular distinction and angiogenic interaction between embryonic arteries and veins revealed by ephrin-B2 and its receptor EphB4. Cell 93: 741-753.

Weinmaster, G. 1997. The ins and outs of Notch signaling. Mol. Cell. Neurosci. 9: 91-102.

Xue, Y., X. Gao, C.E. Lindsell, C.R. Norton, B. Chang, C. Hicks, M. Gendron-Maguire, E.B. Rand, G. Weinmaster, and T. Gridley. 1999. Embryonic lethality and vascular defects in mice lacking the Notch ligand Jagged1. Hum. Mol. Genet. 8: 723-730.

Yamaguchi, T.P., D.J. Dumont, R.A. Conlon, M.L. Breitman, and J. Rossant. 1993. flk-1, an flt-related receptor tyrosine kinase is an early marker for endothelial cell precursors. Development 118: 489-498.

Zimrin, A.B., M.S. Pepper, G.A. McMahon, F. Nguyen, R. Montesano, and T. Maciag. 1996. An antisense oligonucleotide to the notch ligand jagged enhances fibroblast growth factorinduced angiogenesis in vitro. J. Biol. Chem. 271: 3249932502. 


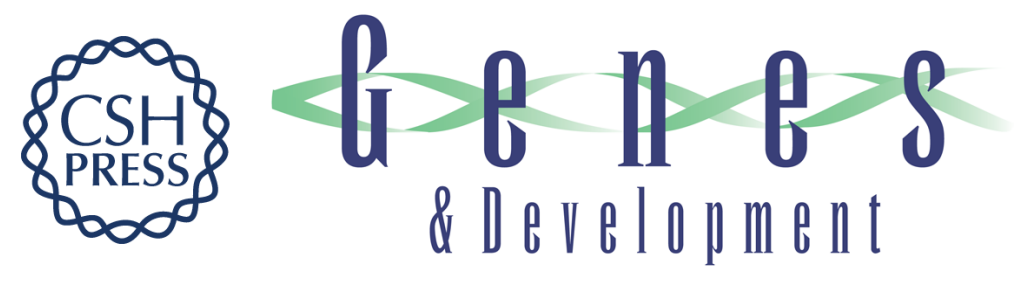

\section{Notch signaling is essential for vascular morphogenesis in mice}

Luke T. Krebs, Yingzi Xue, Christine R. Norton, et al.

Genes Dev. 2000, 14:

Access the most recent version at doi:10.1101/gad.14.11.1343

References This article cites 41 articles, 20 of which can be accessed free at: http://genesdev.cshlp.org/content/14/11/1343.full.html\#ref-list-1

License

Email Alerting Receive free email alerts when new articles cite this article - sign up in the box at the top Service right corner of the article or click here.

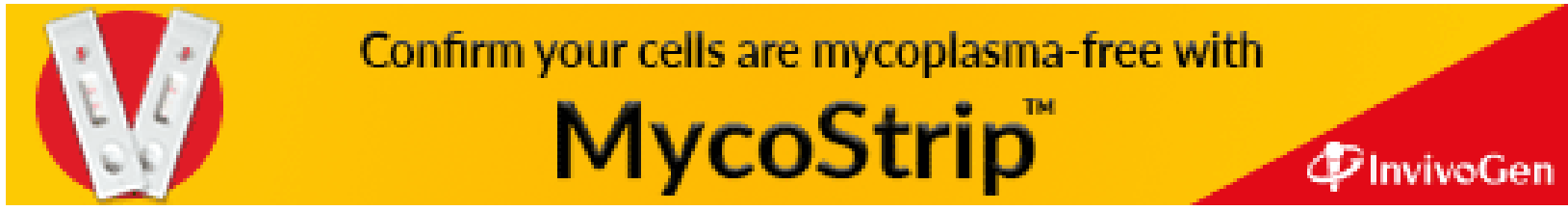

